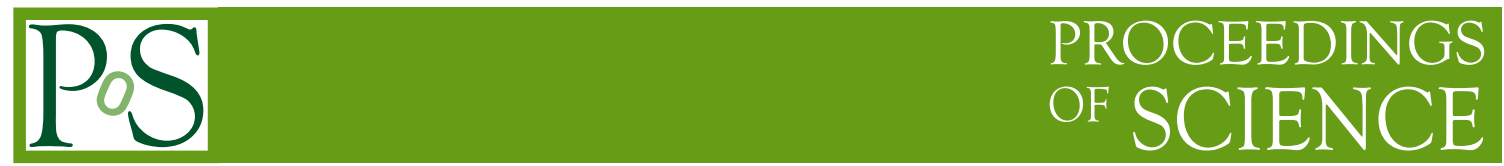

\title{
Wigner Distributions Using Light-Front Wave Functions
}

\section{Asmita Mukherjee*}

Department of Physics, Indian Institute of Technology Bombay; Powai, Mumbai 400076, India

E-mail: asmita@phy.iitb.ac.in

We report on some recent advances in calculating the Wigner distributions for quarks and gluons, using overlaps of light-front wave functions.

QCD Evolution 2017

22-26 May, 2017

Jefferson Lab Newport News, VA - USA

${ }^{*}$ Speaker. 


\section{Introduction}

The most challenging task in hadron physics today is to understand the tomographic picture of the nucleon in terms of quarks and gluons. This leads to the study of the spatial and momentum distributions of these quarks and gluons as well as their spin and angular momentum correlations. In this context, Wigner distributions [1] are objects of intense interest in recent days. Six dimensional Wigner distributions of quarks and gluons in the rest frame of the nucleon were introduced in [2]. In quantum mechanics, Heisenberg's uncertainty principle prevents us from having a join position and momentum space description of a system. So Wigner functions are not positive definite, or they do not have probabilistic interpretation. A five dimensional object in the infinite momentum frame or equivalently in light-front formalism was introduced in [3], which is a function of three momenta and two transverse position coordinates. Taking a Fourier transform with respect to the transverse position $b_{\perp}$ relates the Wigner distributions to the generalized transverse momentum dependent pdfs (GTMDs) [4], which are the so-called mother distributions of the generalized parton distributions (GPDs) and transverse momentum dependent pdfs (TMDs). Some of the Wigner distributions do not have a forward limit related to a known TMD. In fact these are related to the elusive OAM and spin-orbit correlations of quarks and gluons [3,5]. Although there are quite a few theoretical studies to probe the GTMDs in experiments $[6,7,8,9]$, there are so far no experimental data on them. Model calculations of Wigner functions are thus important to get a qualitative idea of them. In this talk, we report on a few recent developments on model calculations of the quark and gluon Wigner distributions [10,11, 12].

\section{Wigner Distributions in a dressed quark model}

We use light-front Hamiltonian formulation [13] in light-cone gauge, using overlaps of LFWFs. The Wigner distributions of quarks are defined as follows: [4, 3]

$$
\rho^{[\Gamma]}\left(b_{\perp}, k_{\perp}, x, s, s^{\prime}\right)=\int \frac{d^{2} \Delta_{\perp}}{(2 \pi)^{2}} e^{-i \Delta_{\perp} \cdot b_{\perp}} W_{s s^{\prime}}^{[\Gamma]}\left(\Delta_{\perp}, k_{\perp}, x\right)
$$

where $b_{\perp}$ is the impact parameter conjugate to $\Delta_{\perp}$, which is the transverse momentum transfer. The quark-quark correlator $W^{[\Gamma]}$ in the above expression can be written as,

$$
W_{s s^{\prime}}^{[\Gamma]}\left(\Delta_{\perp}, k_{\perp}, x\right)=\left.\int \frac{d z^{-} d^{2} z_{\perp}}{2(2 \pi)^{3}} e^{i k . z}\left\langle p^{+}, \frac{\Delta_{\perp}}{2}, s^{\prime}\left|\bar{\psi}\left(-\frac{z}{2}\right) \Omega \Gamma \psi\left(\frac{z}{2}\right)\right| p^{+},-\frac{\Delta_{\perp}}{2}, s\right\rangle\right|_{z^{+}=0}
$$

$P=\frac{1}{2}\left(p^{\prime}+p\right)$ is the average four-momentum of the target state, the momentum transfer in the transverse direction is $\Delta=p^{\prime}-p . s\left(s^{\prime}\right)$ is the helicity of the initial (final) target state. The average four momentum of the active quark is $k$, and $x$ is the longitudinal momentum fraction of the parton. $\Gamma$ is the Dirac matrix and $\Omega$ is the gauge link for color gauge invariance. We use light-cone gauge and take the gauge link to be unity.

The Wigner distribution of the gluon are defined as [4, 3]

$$
\begin{aligned}
x W_{\sigma, \sigma^{\prime}}\left(x, k_{\perp}, b_{\perp}\right) & =\int \frac{d^{2} \Delta_{\perp}}{(2 \pi)^{2}} e^{-i \Delta_{\perp} . b_{\perp}} \int \frac{d z^{-} d^{2} z_{\perp}}{2(2 \pi)^{3} p^{+}} e^{i k . z} \\
& \times\left.\left\langle p^{+},-\frac{\Delta_{\perp}}{2}, \sigma^{\prime}\left|\Gamma^{i j} F^{+i}\left(-\frac{z}{2}\right) F^{+j}\left(\frac{z}{2}\right)\right| p^{+}, \frac{\Delta_{\perp}}{2}, \sigma\right\rangle\right|_{z^{+}=0}
\end{aligned}
$$


Suppressing the color indices, we have,

$$
F^{+i}=\partial^{+} A^{i}-\partial^{i} A^{+}+g f^{a b c} A^{+} A^{i}
$$

The operator structure for gluon at twist two are [14] (i) $\Gamma^{i j}=\delta_{\perp}^{i j}$, (ii) $\Gamma^{i j}=-i \varepsilon_{\perp}^{i j} \quad$ (iii) $\Gamma^{i j}=$ $\Gamma^{R R}$ and (iv) $\Gamma^{i j}=\Gamma^{L L}$, where $L(R)$ are left(right) polarization of the gluon. Wigner distribution for gluons need two gauge links for color gauge invariance, which we have not shown explicitly in the above expression. We use light-cone gauge and take the gauge links to be unity. $\sigma$ and $\sigma^{\prime}$ are the helicities of the target state. Instead of a proton target, we take the state to be a quark dressed with a gluon at one loop in perturbation theory. This can the thought of as a field theory inspired perturbative model having a gluonic degree of freedom. The quark has non-zero mass and both quark and gluon have non-zero transverse momenta. A dressed quark state can be expanded in Fock space as

$$
\begin{aligned}
\left|p^{+}, p_{\perp}, s\right\rangle= & \Phi^{s}(p) b_{s}^{\dagger}(p)|0\rangle+\sum_{s_{1} s_{2}} \int \frac{d p_{1}^{+} d^{2} p_{1}^{\perp}}{\sqrt{16 \pi^{3} p_{1}^{+}}} \int \frac{d p_{2}^{+} d^{2} p_{2}^{\perp}}{\sqrt{16 \pi^{3} p_{2}^{+}}} \sqrt{16 \pi^{3} p^{+}} \delta^{3}\left(p-p_{1}-p_{2}\right) \\
& \times \Phi_{s_{1} s_{2}}^{s}\left(p ; p_{1}, p_{2}\right) b_{s_{1}}^{\dagger}\left(p_{1}\right) a_{s_{2}}^{\dagger}\left(p_{2}\right)|0\rangle
\end{aligned}
$$

The two-particle light-front wave function (LFWF) is written in terms of the boost-invariant LFWF as

$$
\sqrt{P^{+}} \Phi\left(p ; p_{1}, p_{2}\right)=\Psi\left(x_{i}, q_{i}^{\perp}\right)
$$

This can be calculated in light-front Hamiltonian perturbation theory and is given by:

$$
\begin{aligned}
\Psi_{s_{1} s_{2}}^{s a}\left(x, q^{\perp}\right) & =\frac{1}{\left[m^{2}-\frac{m^{2}+\left(q^{\perp}\right)^{2}}{x}-\frac{\left(q^{\perp}\right)^{2}}{1-x}\right]} \frac{g}{\sqrt{2(2 \pi)^{3}}} T^{a} \chi_{s_{1}}^{\dagger} \frac{1}{\sqrt{1-x}} \\
& \times\left[-\frac{2 q^{\perp}}{1-x}-\frac{\left(\sigma^{\perp} \cdot q^{\perp}\right) \sigma^{\perp}}{x}+\frac{i m \sigma^{\perp}(1-x)}{x}\right] \chi_{s}\left(\varepsilon_{s_{2}}^{\perp}\right)^{*}
\end{aligned}
$$

We here use two-component formalism [15], $\chi, T^{a}, m$ and $\varepsilon_{s 2}^{\perp}$ are the two-component spinor, color $\mathrm{SU}(3)$ matrices, mass of the quark, and polarization vector of the gluons, respectively.

The Wigner distributions for different combinations of polarizations of the target and the probed quark or gluon are expressed in terms of overlaps of the light-front wave functions [10]. The single particle sector of the Fock space plays an important role when $x=1$ through the wave function renormalization. Wigner distributions are calculated analytically using LFWFs and the Fourier transform with respect to $\Delta_{\perp}$ is done numerically. The analytic results for all the quark and gluon Wigner distributions for a simple composite spin $1 / 2$ system can be found in $[10,11]$. The full calculation of the Wigner distributions and spin-spin correlations in quark-diquark model, where the LFWF is modeled by Ads/QCD correspondence, can be found in [12]. Here we report on some of our numerical results. 


\section{Numerical Results}

The Wigner distributions are functions of five variables, two transverse position $b_{\perp}$, two transverse momenta $k_{\perp}$ and one longitudinal momentum fraction for the quark(gluon) $x$. In the plots we integrate them over $x$. For our numerical calculations, we use a better integration strategy called the Levin method [16], which suits our oscillatory integrands, improving the convergence of the results with respect to the change in the upper limit of the $\Delta_{\perp}$ integration as was seen in the earlier calculation $[17,18]$. We have used the upper limit of the $\Delta_{\perp}$ integration to be $\Delta_{\max }=20 \mathrm{GeV}$, and the results are independent of this cutoff. We have taken the mass of the quark to be $m=0.33 \mathrm{GeV}$. For all plots in $b_{\perp}$ space, we took a fixed value of $k_{\perp}$ and vice versa.

Fig. 1(a) shows the plot of $\rho_{U U}$ in $b$ space, which is the distribution of an unpolarized quark in an unpolarized target state. Fig. 1(b) shows the plot of $\rho_{U L}$ in $b$ space. For a dressed quark, $\rho_{U L}=\rho_{L U}$. This Wigner distribution is related to the orbital angular momentum of the quark. A dipole structure similar to chiral quark soliton model and constituent quark model [3] is seen. In Fig. 2(a) we have plotted $\rho_{U T}^{x}$, which gives the distribution of transversely polarized quark in an unpolarized target; quark polarization in the $x$ direction. Here we also observe a dipole structure. TMD limit of $\rho_{U T}$ is the Boer-Mulders function. As we have taken the gauge link to be unity, we cannot access the T-odd distributions like the Boer-Mulders function. The behavior in $b$ space is similar to spectator model [19]. In Fig. 2(b) we have shown $\rho_{L T}^{x}$ in $k_{\perp}$ space. These distributions describe a transversely polarized quark in a longitudinally polarized target state and here the direction of the polarization of the quark is in the $x$-direction. We observe a dipole structure in $k$-space. The TMD limit of this is related to the worm-gear function, $h_{1 L}^{\perp}$.

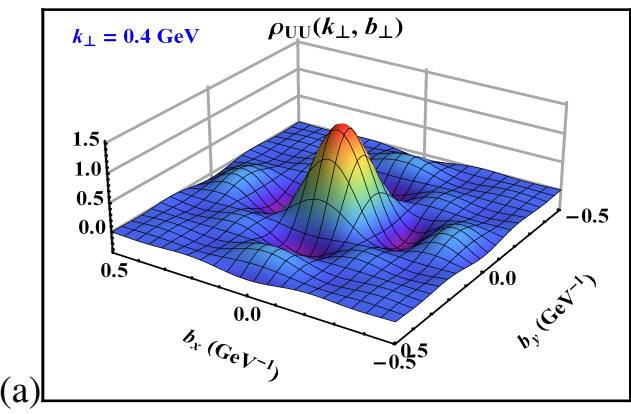

(b)

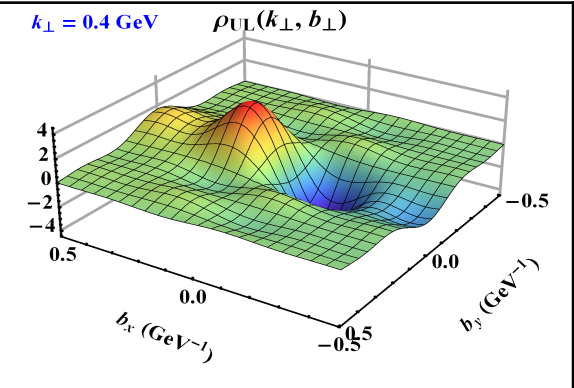

Figure 1: 3D plots of Wigner distributions $\rho_{U U}\left(\mathbf{k}_{\perp}, \mathbf{b}_{\perp}\right)$ and $\rho_{U L}\left(\mathbf{k}_{\perp}, \mathbf{b}_{\perp}\right)$ at $\Delta_{\max }=20 \mathrm{GeV}$ in $b$ space [10].

In Fig. 3(a) we have plotted the gluon Wigner distribution $W_{U U}$, which is the distribution of an unpolarized gluon in an unpolarized dressed quark. As discussed above, each gluon Wigner distribution needs two gauge links for color gauge invariance. Depending on whether it is a ++ or +gauge link combination, it is called a Weizsacker-Williams type or dipole type gluon distribution. In [7] it was shown that both of them give the same orbital angular momentum distribution of the gluon. In our calculation, we have taken the gauge links to be unity in light-cone gauge. The gluon distribution has a positive peak at the center of the $b$ space. Fig. 3 (b) shows $W_{U L}$, which is the Wigner distribution for a longitudinally polarized gluon in an unpolarized target state in $b_{\perp}$ space. This shows dipolelike structure. Note that the behavior of $W_{U U}$ near $b_{\perp}=0$ is determined by the 
(a)

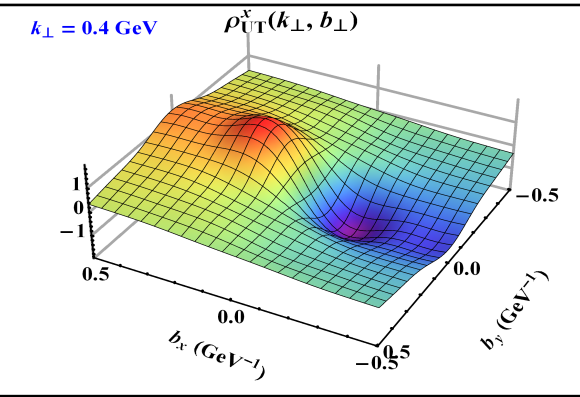

(b)

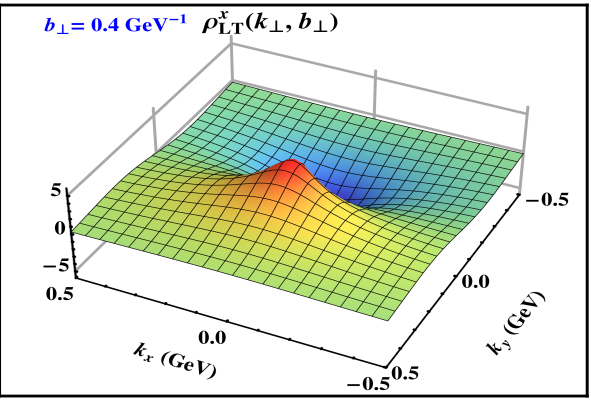

Figure 2: 3D plots of Wigner distributions $\rho_{U T}^{x}\left(\mathbf{k}_{\perp}, \mathbf{b}_{\perp}\right)$ in $b$ space and $\rho_{L T}^{x}\left(\mathbf{k}_{\perp}, \mathbf{b}_{\perp}\right)$ in $k$ space at $\Delta_{\max }=$ $20 \mathrm{GeV}[10]$.

relative dominance of the $k_{\perp}^{2}$ and $\Delta_{\perp}^{2}(1-x)^{2}$ terms in the numerator. As $\Delta_{\text {max }}$ increases, the second term dominates over the first, as a result, the peak at $b_{\perp}=0$ becomes positive.

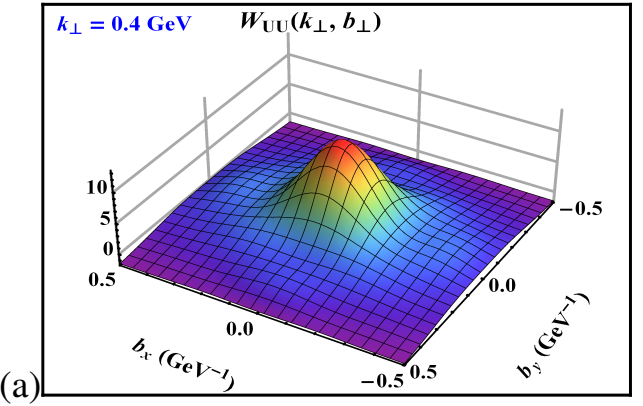

(b)

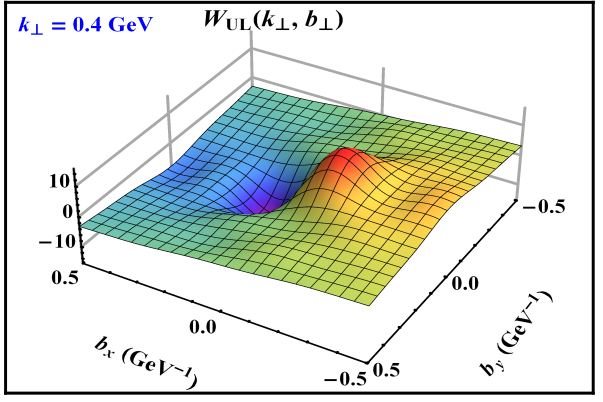

Figure 3: 3D plots of gluon Wigner distributions $W_{U U}$ and $W_{U L}$ in $b$ space for $\Delta_{\max }=20 \mathrm{GeV}$ [11].

In Fig. 4(a) and 4(b) we have shown the Wigner distributions $\rho_{U U}$ and $\rho_{U L}$ respectively for the $\mathrm{u}$ quark in a quark-diquark model for the proton, modeled using a modified form of LFWF obtained using Ads/QCD prediction [12]. Here also, we have integrated over $x$. Average quadrupole distortion for $\rho_{U U}$ is found to be zero in this model. Unlike other models, no favored direction is there in $b$ space, and the distribution is circularly symmetric. $\rho_{U L}$ has a dipole structure. The OAM for $u$ quarks is antiparallel to quark spin, same as in scalar diquark model and opposite to that observed in constituent quark model.

\section{Conclusion}

We presented some recent advances in the calculation of the Wigner distributions of quarks in dressed quark target model and in a quark-diquark model for the proton using a modified LFWF obtained using Ads/QCD. The dressed quark target represents a composite spin 1/2 state with a gluonic degree of freedom, and we have calculated the gluon Wigner distributions as well. These are expressed in terms of overlaps of LFWFs and using their analytic form we have calculated 
(a)

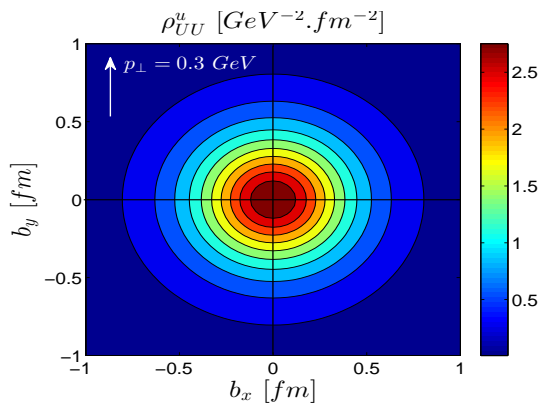

(b)

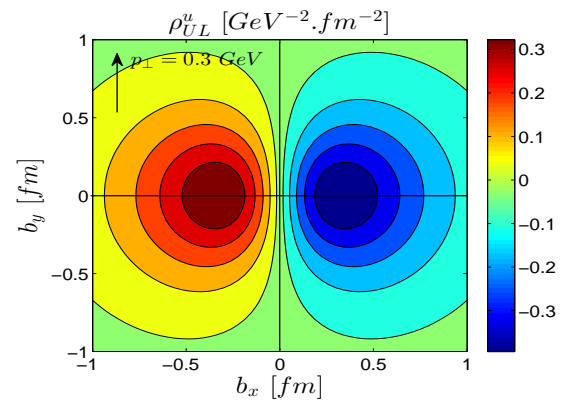

Figure 4: Plots of $u$-quark Wigner distributions $\rho_{U U}$ and $\rho_{U L}$ in $b$ space in light-front quark-diquark model [12].

them. Better convergence of the results is obtained using Levin's method of integration. Further work would involve inclusion of the gauge link in their description.

\section{Acknowledgement}

AM thanks the organizers of the QCD Evolution Workshop at Jefferson Lab for the invitation and support. J. More, S. Nair, D. Chakrabarti, C. Mondal and T. Maji are thanked for collaboration.

\section{References}

[1] E. P. Wigner, Phys. Rev. 40, 749 (1932).

[2] X. D. Ji, Phys. Rev. Lett. 91, 062001 (2003).

[3] C. Lorce and B. Pasquini, Phys. Rev. D 84, 014015 (2011).

[4] S. Meissner, A. Metz and M. Schlegel, J. High Energy Phys. 08 (2009) 056.

[5] Y. Hatta, Phys. Lett. B 708, 186 (2012).

[6] Y. Hatta, B-W. Xiao, F. Yuan, Phys.Rev. D 95, (2017) no.11, 114026.

[7] Y. Hatta, Y. Nakagawa, F. Yuan, Y. Zhao, Phys.Rev. D 95, (2017) no.11, 114032.

[8] Y. Hagiwara, Y. Hatta, T. Ueda, Phys. Rev. D 94, No. 9, 094036 (2016).

[9] S. Bhattacharya, A. Metz, J. Zhou, Phys. Lett. B 771396 (2017).

[10] J. More, A. Mukherjee, S. Nair, Phys. Rev. D 95, No. 7, 074039 (2017).

[11] J. More, A. Mukherjee, S. Nair, arXiv:1709.00943 [hep-ph].

[12] D. Chakrabarti, T. Maji, C. Mondal and A. Mukherjee, Phys. Rev. D 95, No. 7, 074028 (2017).

[13] A. Harindranath, R. Kundu and W. M. Zhang, Phys. Rev. D 59, 094012 (1999); Phys. Rev. D 59, 094013 (1999).

[14] C. Lorce and B. Pasquini, J. High Energy Phys. 09 (2013) 138.

[15] W. M. Zhang and A. Harindranath, Phys. Rev. D 48, 4881 (1993). 
[16] D. Levin, Math. Comp. 38, 531 (1982).

[17] A. Mukherjee, S. Nair and V. K. Ojha, Phys. Rev. D 90, 014024 (2014).

[18] A. Mukherjee, S. Nair and V. K. Ojha, Phys. Rev. D 91, 054018 (2015).

[19] T. Liu and B. Q. Ma, Phys. Rev. D 91, 034019 (2015). 